\title{
Abstracts
}

\section{Abstracts selected through 999 EMS Research Forum peer review process and presented orally or by poster at Ambex 2004}

\section{Oral presentations}

\author{
WINNER OF THE EMERGENCY MEDICINE JOURNAL \\ (EMJ) AWARDED FOR MOST ORIGINAL RESEARCH
}

\section{$00110 \%$ OR $50 \%$ DEXTROSE IN THE TREATMENT OF HYPOGLYCAEMIA OUT-OF-HOSPITAL? A RANDOMISED CONTROLLED TRIAL}

C. Moore, M. Woollard. Pre-hospital Emergency Research Unit, Welsh Ambulance Services NHS Trust, Lansdowne Hospital, Cardiff, UK

Objective: This randomised controlled trial aimed to determine whether $10 \%$ dextrose is as safe and effective as $50 \%$ dextrose when treating hypoglycaemia out-of-hospital.

Methods: Hypoglycaemic patients in the out-of-hospital setting with a blood glucose level of $4 \mathrm{mmols} / \mathrm{l}$ or less were randomised to receive either intravenous $10 \%$ or $50 \%$ dextrose. Paramedics in a large UK ambulance service administered either concentration of dextrose in 5 gram increments. $10 \%$ dextrose was administered in $50 \mathrm{ml}$ aliquots via a three-way tap and giving set and $50 \%$ in $10 \mathrm{ml}$ aliquots from pre-filled $(25 \mathrm{~g}$ in $50 \mathrm{ml}$ ) syringes. The maximum cumulative dose for subjects in both groups was limited to $25 \mathrm{~g}$. Paramedics recorded standard pre and post-treatment clinical data and rated the convenience of administration of each dextrose formulation using a Lickert scale.

Results: Fifty one patients were recruited $(10 \% n=25,50 \% n=26)$. There were no significant differences between groups in age or sex profile, median pretreatment Glasgow Coma Score (GCS) and blood glucose level, or the proportion of insulin dependent diabetics. Following treatmen there were no significant differences in median time to recovery (8 minutes), median post-treatment GCS (15), subjects experiencing a further hypoglycaemic episode within 24 hours ( 4 per group), time on scene, or ease of administration. There were, however, significant differences in the median total dose of dextrose administered $(10 \%=10 \mathrm{grams}$, $50 \%=25$ grams, $p<0.001$ ) and median post-treatment blood suga levels $(10 \%=6.2 \mathrm{mmols} / \mathrm{l}$ and $50 \%=9.4 \mathrm{mmols} / \mathrm{l}, \mathrm{p}=0.003)$. There were no reports of extravasation injury from either group.

Conclusions: $10 \%$ dextrose proved as effective as $50 \%$ in the out-ofhospital treatment of hypoglycaemia. It is administered in lower doses than a $50 \%$ presentation, which results in more acceptable posttreatment blood glucose levels.

\section{AMBULANCE INITIATIVES TO REDUCE EMERGENCY DEPARTMENT ATTENDANCE}

M. W. Cooke, J. Fisher, J. Dale, I. Todd. Warwick Emergency Care and Rehabilitation, Warwick Medical School, UK

Studies have shown that $40 \%$ of emergency ambulance calls do not require an emergency response and yet many are still transported to the ED.

Aims: To review evidence on prehospital initiatives to reduce ED attendance

Methods: A systematic literature review has been undertaken, according to NHS Centre for Reviews and Dissemination guidelines, to determine innovations to reduce waits in emergency departments. The review includes explicit criteria for establishing search strategies in electronic databases, journal web sites, hand searching, and personal request. Papers were reviewed and quality assessed. This paper describes those papers relating to the ambulance interventions to reduce emergency department attendances.

Results: The literature describes two key interventions. 1. Systems to divert emergency ambulance calls to nurse advice systems. 2 . Ambulance crews discharging patients or transporting them elsewhere than the emergency department.
It is possible to predict calls which may only need advice but with varying accuracy. In the largest RCT 9\% of those thought to need advice were eventually admitted to hospital.

The potential for discharging from the scene is great (up to $40 \%$ of calls) but the safety has not been proven. Present dispatch systems and triage systems are not sufficiently sensitive. Note reviews have revealed high numbers (up to $25 \%$ ) of critical cases assigned to discharge groups. Trials have shown minimal effect and had high (up to 9\%) incorrect discharge rates.

Conclusions: Present evidence has severe limitations but does not support the safety or effectiveness of diverting emergency ambulance calls or paramedics discharging from the scene. The full literature review will be available via www.waits.info.

\section{WHAT ARE THE BELIEFS AND ATTITUDES OF PARAMEDICS TO PREHOSPITAL THROMBOLYSIS? A QUESTIONNAIRE STUDY}

A. Walker, J. Humphrey, T. B. Hassan. Pontefract General Infirmary, UK

Background: The Ambulance Services have a critical role in the management of acute myocardial infarction. The Coronary Heart Disease NSF proposed thrombolysis targets and encouraged extending the paramedic role in managing AMI. To our knowledge there has been no research carried out questioning individual paramedics to ascertain their views of paramedic delivered prehospital thrombolysis (PHT).

Methods: A postal questionnaire sent to the 250 paramedics in West Yorkshire Metropolitan Ambulance Service (WYMAS) who could perform and interpret a 12 lead ECG at the time of the study. The questionnaire included the beliefs of risks and benefits of $A M I$ treatments, questions on the ability of paramedics to carry out PHT and opinions regarding this future role.

Results: 193 paramedics replied (77\%). 83\% paramedics questioned felt paramedics would be capable to deliver PHT, $67 \%$ felt thrombolysis was safe for use by paramedics, only $12 \%$ felt paramedics should not give PHT at all.

Autonomous paramedic PHT $(42 \%)$ and telemetry with physician directed PHT $(46 \%)$ were both supported. They wanted hospital-based training $(80 \%)$, and a nationally recognised thrombolysis training certificate for paramedics (96\%). There were concerns regarding the risks of thrombolysis, with overestimates of the risks of extra deaths (71\%), and bleeding (90\%). 95\% felt there should be additional pay for the extra responsibility; some felt thrombolysis training should be optional. There was fear of litigation, personal liability for clinical incidents and concern around support systems.

Conclusion: The majority of paramedics supported the principle of PHT; their specific concerns are the risks of thrombolytic treatment, training, and medico-legal implications for them as individual paramedics. The training for this role should therefore include evidence-based medical education, in-hospital training, and reassurance regarding medico-legal issues. Models for paramedic thrombolysis for each ambulance service should include the views of paramedics.

\section{EMERGENCY CARE PRACTITIONERS IN LONDON - A QUALITATIVE VIEW OF THEIR DEVELOPMENT}

M. Halter' ', K. Start' ${ }^{2}$ ' London Ambulance Service NHS Trust, UK; ${ }^{2}$ University of Kingston, UK

Introduction: The role of Emergency Care Practitioner (ECP) is developing around the UK ECPs are training for a role with greater autonomy and this has been encouraged in London Ambulance Service through education, clinical training, and project roles. There is no published evidence about ECPs. This paper reports $\mathrm{ECPs}^{\prime}$ views on their development. 
Method: All ECPs $(n=22)$ in post at the time were asked to participate in a semi-structured interview, the guide of which was drawn up from earlier team away days.

Thematic content analysis was carried out.

Results: 21 ECPs were interviewed over a 6 week period. ECPs reported mixed experiences. All reported positively on education content, but saw supervision and mentorship as necessary to translate theory into practice and achieve competence. Views differed on these requirements. Confidence differed between ECPs, individually, but also in cohorts - early in the education package confidence dips (recognising current knowledge limits) and appears to increase with clinical mentorship, and with practice. All ECPs with allocated project roles, including negotiating with Primary Care Trusts or ambulance service colleagues, described this as difficult; for some this was overwhelming, for others the experience contributed to learning. Some loss of identity was detected with a shift from belonging to the ambulance service to working alone or in other environments. For some it was early days and frustration associated with an evolving project continued; others cited attitude, practice and personal changes - thinking broadly about the patient's needs and treatment possibilities, and working with the wider health team, described as a shift from an emergency to a primary care attitude.

Study limitation: The interviewer is an in-house researcher, with possible questioning bias.

Conclusions: ECPs are enhancing their educational knowledge and clinical practice and taking responsibility for project direction. This is challenging for the majority, and support is required in their move toward greater responsibility and autonomy.

\section{Posters}

\section{WINNER OF THE EMERGENCY MEDICINE JOURNAL (EMJ) AWARDED FOR BEST POSTER}

\section{USE OF AN ACTIVE COMPRESSION DECOMPRESSION DEVICE IN PREHOSPITAL CARDIAC ARREST BY AMBULANCE PARAMEDICS AND AMBULANCE TECHNICIANS}

\section{S. Box. Dorset Ambulance NHS Trust, UK}

Introduction: The active compression decompression device the AMBU CARDIO PUMP has just been introduced to the UK prehospital market. This study used the AMBU- CARDIO PUMP to assess the effects of active compression decompression CPR compared with standard CPR in the prehospital environment.

Method: Patients who suffered a cardiac arrest in the Bournemouth conurbation were randomly assigned to receive either active compression decompression CPR or standard CPR depending on which crew attended the arrest. The primary study end point was arrival at a designated Accident and Emergency Department, with a secondary end study point of 4 weeks post arrest.

Results: Short term outcome among patients assigned to standard CPR and active compression decompression CPR

Both the ACD-CPR and standard CPR groups ended up with similar numbers in each group, with each group being $66 \%$ male. The initial cardiac rhythm of VF occurred in $41 \%$ of the ACD-CPR group against $58 \%$ for standard CPR. At the primary end study point $66 \%$ of the patients treated with ACD-CPR had return of spontaneous circulation compared to $33 \%$ for standard CPR. Survival at 4 weeks was measured at $33 \%$ for the ACD-CPR with none of the standard CPR group surviving past 7 days.

\begin{tabular}{|c|c|c|}
\hline & $\begin{array}{l}\text { STANDARD CPR } \\
\mathrm{N}=12\end{array}$ & $\begin{array}{l}\text { ACD CPR } \\
N=12\end{array}$ \\
\hline Return of spontaneous circulation & $33 \%$ & $66 \%$ \\
\hline Survival at one hour & $33 \%$ & $58 \%$ \\
\hline Admission to ITU & $33 \%$ & $58 \%$ \\
\hline Survival at 24 hours & $8 \%$ & $50 \%$ \\
\hline Survival at 7 days & $8 \%$ & $41 \%$ \\
\hline Survival at 4 weeks & $0 \%$ & $33 \%$ \\
\hline
\end{tabular}

Conclusions: Active compression decompression CPR using the AMBU-CARDIO PUMP performed during advanced life support appears to have significantly improved survival rates among patients who have a pre hospital cardiac arrest.

The author recognises that this is small-scale research and the findings would suggest that further research into active compression decompression CPR is indicated.

\section{WINNER OF THE JOINT ROYAL COLLEGES AMBULANCE LIAISON COMMITTEE (JRCALC) AWARD FOR RESEARCH MOST LIKELY TO AFFECT PRACTICE}

\section{A MULTI-CENTRE EVALUATION OF MANAGING LOW PRIORITY 999 CALLS BY NURSE ADVICE - PROGRESS SO FAR}

J. Turner', H. Snooks' ${ }^{2}{ }^{1}$ Medical Care Research Unit, University of Sheffield, UK; ${ }^{2}$ The Clinical School, University of Wales, Swansea, UK

Study Aim: The aim of the study is to assess the clinical, service and cost effectiveness of transferring some non-urgent 999 ambulance calls to NHS Direct nurse advisers for further assessment and management.

Methods: The study has been designed using a mixed randomised controlled trial (RCT) and controlled observational study approach. There are 2 stages:

Stage 1 - is a randomised controlled trial. Over a 12 month period individuals calling 999 and triaged by the ambulance control call taker to one of the study EMD codes are randomly allocated to receive further assessment by the nurse adviser or dispatch of the standard response of a paramedic ambulance after consenting to participate. These callers are then sent a postal questionnaire to elicit what action they took and their satisfaction with the service received. Comparisons will be made between intervention and control groups to determine if the service change provides a clinically effective and acceptable service.

Stage 2 - Is a controlled observational study. Over a 4 month period all calls with appropriate EMD codes will be passed to nurse advisers to identify the practical and operational effects of the service change. The cost consequences of the service will also be measured.

Practicalities: The practical implications of setting up and conducting a research trial in an operational environment can be difficult. Strategies to overcome these have included:

- Obtaining patient consent - Consent has to be obtained verbally at the time of the 999 call. A pre-consent randomisation design has been used to simplify this process and minimise confusion and the amount of explanation required. Simple consent protocols for EMDs and nurses have been developed and a standardised recruitment algorithm provided to each service.

- Randomisation procedures - For Omega calls an electronic randomisation programme has been built in to the AMPDS system. In Manchester, which uses Alpha calls, EMD stations are randomly assigned to intervention or control group each day. EMDs refer identified calls to the appropriate response for that day.

- Developing referral processes - In Manchester a seamless, integrated call transfer process has been developed. The other sites refer to a remote NHS Direct centre where calls are directed straight to a nurse identified to accept ambulance service calls and bypassing the initial NHS Direct call handling process.

- Gaining staff engagement and co-operation in the project-A project board involving the research team, senior managers from both ambulance and NHS Direct services, EMDs and nurses was set up before the project started. All members have been fully involved in setting up and developing the practical components of consent and recruitment procedures, referral processes and data collection. The group meets regularly to review progress, identify problems and share experiences and best practice.

Progress so far: The RCT started in Autumn 2003. The Two Shires, Oxfordshire and Welsh ambulance service are testing AMPDS Omega codes and transfer calls to an NHS Direct call centre for nurse advice. Greater Manchester Ambulance Service are testing AMPDS Alpha codes and nurse advice is provided in the control room.

To date 1583 calls have been randomised to the intervention group and 1847 to the control group. About one third of these calls have given consent for further follow up. A high proportion of AMPDS Alpha calls are returned to the ambulance service $(67 \%)$ whereas only $35 \%$ of Omega calls are returned at this point in the study. 
007 SECURING THE PREHOSPITAL AIRWAY: A COMPARISON OF PARAMEDIC AIRWAY MANAGEMENT USING THE DISPOSABLE LARYNGEAL MASK AND ENDOTRACHEAL TUBE

C. D. Deakin, R. Peters, P. Tomlinson, M. Cassidy. Hampshire Ambulance Service NHS Trust, UK

Introduction: Prehospital endotracheal intubation is known to be associated with significant prehospital morbidity. The recent introduction of a disposable laryngeal mask airway has provided paramedics with an alternative to endotracheal intubation. The time taken to secure the airway and ventilate the patient by paramedics was compared using the two devices in patients undergoing elective surgery.

Methods: Patients undergoing general anaesthesia for cardiac surgery were studied. Paramedics trained in laryngeal mask use and endotracheal intubation participated in the study. A Portex single use Soft Seal ${ }^{T M}$ Laryngeal Mask (Smiths Medical, Hythe, UK) was inserted and removed, followed by a Portex endotracheal tube. Time taken from beginning of the procedure to ventilation of the patient was recorded. Cricoid pressure was applied as appropriate.

Results: Laryngeal mask insertion and endotracheal intubation was attempted on 52 patients. Median age was 63.5 years (range 39-83 years). Laryngeal mask insertion was successful in $88.5 \%(46 / 52)$ patients; endotracheal intubation was successful in $71.2 \%(37 / 52)$ patients (after no more than 2 attempts), $p=0.049$. Intubation success was related to laryngoscopic view $187.5 \%$ grade $1,56.3 \%$ grade 2 , $0.0 \%$ grade $3 . p<0.001)$. When laryngeal mask/endotracheal tube insertion were both successful $(n=35 / 52)$, there was no significant difference in median time to secure the airway (LM 47.0 secs (range 24$126 \mathrm{sec}$ ) $\vee$ ETT 52.0 secs (range $27-148$ secs) $p=0.22$ ). Laryngeal mask insertion was successful in $80.0 \%(12 / 15)$ patients in whom endotracheal intubation had failed.

Discussion and Conclusion: Even under optimal conditions, 30\% of attempts at intubation by paramedics were unsuccessful. A disposable laryngeal mask has a higher success rate in securing the airway and overall, secures the airway more reliably than endotracheal intubation.

\section{IDENTIFYING THE SERIOUSLY ILL - AMPDS V CATEGORY A}

A. Heward. London Ambulance Service NHS Trust, UK

Introduction: AMPDS has been used in the UK since the late 1990's, following recommendations from the Department of Health. AMPDS is an American system designed to identify the response need of the patient (Paramedic $v$ Basic Life Support). This system is currently used in 13 countries and seen as an international standard of care. In the UK, the Department of Health imposed a different working regime for the system to operate within, explicitly identifying codes that ambulance services must respond to within eight minutes regardless of the response need identified by AMPDS

This study looks at the accuracy of these two methods to assess which identifies the most seriously ill patients.

Methods: An observational study was undertaken studying one years data (662 396 emergency activations) that were triaged using AMPDS version 10.4

Data were analysed for AMPDS determinant and patient outcome (cardiac arrest or hospital pre-alert), which were compared to AMPDS response need and the Department of Health Category A Dataset.

Findings: AMPDS identified $97 \%$ of Cardiac Arrests as requiring a Paramedic response whilst the Category A dataset identified just $85 \%$ as needing a response within eight minutes

AMPDS identified over $91 \%$ of calls resulting in a hospital pre-alert as requiring a paramedic whilst Category A Dataset identified just 35\%.

Conclusion: AMPDS is, from this data, more accurate at identifying seriously ill patients than the Department of Health issued Category A dataset. This would indicate that the Category A dataset is neither as refined nor as accurate as AMPDS. Lessons must be learnt from the accuracy of AMPDS and the dataset refined in line with this information.

\section{DOES THE ADVANCED MEDICAL PRIORITY DESPATCH SYSTEM (AMPDS) EFFECTIVELY IDENTIFY PATIENTS WITH AN ACUTE CORONARY SYNDROME?}

C. D. Deakin, D. M. Sherwood, N. Mortimer, A. Smith, M. Cassidy. Hampshire Ambulance Service NHS Trust, UK

Introduction: The National Service Framework for Coronary Heart Disease requires identification of patients with an acute coronary syndrome (ACS) to enable prompt delivery of prehospital thrombolysis
Identification of these patients is made through the Advanced Medical Priority Despatch System (AMPDS), which is now the common triage tool for '999' calls in the UK. Accurate identification of these patients is necessary to enable efficient use of resources.

Methods: All ' 999 ' calls to Hampshire Ambulance Service NHS Trust (HAST) from the Southampton and Portsmouth areas over a 2 month period (Jan-Feb 2004) were analysed. Patients classified as chest pain by v10.4 AMPDS (Card 10) were specifically identified. Myocardial Infarct National Audit Project (MINAP) data was obtained from acute hospitals in the Portsmouth and Southampton areas to identify the actual number of these patients with a true ACS.

Results: 13411 ' 999 ' calls were made to HAST from the Southampton and Portsmouth areas. Of these, 1118 patients were categorised as having chest pain, but only 61 of these were subsequently diagnosed as having an ACS. Twenty one patients not diagnosed with chest pain were subsequently determined to have an ACS. Sensitivity of AMPDS for detecting ACS in this sample was $74.4 \%$ and specificity $91.6 \%$. Positive predictive value was $5.2 \%(95 \% \mathrm{Cl} 4.1$ to $6.6 \%)$. Thirty four percent (28/ 82) of patient with confirmed ACS were classified as non-life threatening incidents (LTI) by AMPDS (24.6\% (15/61) of those with chest pain, $61.9 \%(13 / 21)$ of those without chest pain).

Discussion and Conclusion: Only one of approximately every 20 patients with chest pain has an acute coronary syndrome. One third of ACS patients were not categorised to receive an 8 minute response. AMPDS is a poor predictor in identifying patients with ACS and therefore of limited use when matching resources to the clinical needs of these patients.

\section{0 'NO SEND' POLICY: INTERIM EVALUATION OF IMPLEMENTATION AT THE LONDON AMBULANCE SERVICE}

S. Johnson, M. Halter, D. Treleaven. London Ambulance Service NHS Trust, UK

Introduction: An emergency ambulance response to patients whose condition is not serious may not be required. Clinical telephone advice (CTA) can be acceptable. Some patients continue to request an ambulance. A 'No Send' policy informs patients that an ambulance will not be sent, i.e. refused. Early implementation results are presented.

Aims and methods of the evaluation:

\begin{tabular}{ll|}
\hline Abstract 010 & Method \\
\hline Aim & $\begin{array}{l}\text { All patients contacted and telephone } \\
\text { questionnaire administered 7- } \\
10 \text { days following the } 999 \text { call } \\
\text { To gain a view on acceptability } \\
\text { to patients }\end{array}$ \\
$\begin{array}{ll}\text { To identify and review adverse } \\
\text { incidents }\end{array}$ & $\begin{array}{l}\text { A\&E senior medical staff where the } \\
\text { patient reported dissatisfaction and a } \\
\text { poor outcome } \\
\text { Consultation with the Patients Forum }\end{array}$ \\
$\begin{array}{l}\text { To gain a view on acceptability } \\
\text { to the public } \\
\text { To assess staff compliance to } \\
\text { procedure }\end{array}$ & $\begin{array}{l}\text { Quality Assurance (QA) } \\
\text { To assess staff confidence in } \\
\text { using the procedure }\end{array}$ \\
$\begin{array}{l}\text { Semi structured interview with 50\% } \\
\text { sample of CTA staff trained to } \\
\text { implement 'no send' } 2 \text { months after } \\
\text { policy start }\end{array}$ \\
$\begin{array}{l}\text { Review of number of calls invoked } \\
\text { ambulance resource use }\end{array}$ & $\begin{array}{l}\text { against number of ambulance } \\
\text { responses in the appropriate } \\
\text { prioritisation codes }\end{array}$ \\
\hline
\end{tabular}

Results: 249 'no send' cases were identified in 5 months. Many presenting complaints were minor traumatic injury and laceration to limb extremities.

$167(67 \%)$ patients responded to the questionnaire. $155(93 \%)$ stated they understood the advice given. 128 (77\%) followed the advice. 121 (72\%) expressed satisfaction.

18 patients called 999 again within 7 days, with some receiving an ambulance transfer to $\mathrm{A} \& \mathrm{E}$.

Thirteen other calls were potential adverse incidents, with agreement that seven should have received an ambulance. Review highlighted poor communication, and some clinical knowledge limitations. 
The Patients' Forum suggested support for the policy.

QA identified use of 'excessive demand on ambulance resources' as a reason for not sending, and non-use of the policy in some conditions.

Although most staff welcomed the introduction of the policy, concerns exist and staff prefer to persuade the patient rather than refuse.

The policy is responsible for $15 \%$ of CTA cases where an ambulance was not sent.

Study limitations:

- No clinical follow-up

- Small numbers of clinical reviews to date; differences of reviewer opinion

- No comparator data.

Conclusions: These early results are more positive than expected on patient satisfaction when refusing an ambulance; however, adverse incidents, clinical review, QA and staff confidence highlight issues that provoke concern and suggest caution.

\section{DISTRICT NURSE AND PARAMEDIC RESPONSE TEAM PILOT - LONDON AMBULANCE SERVICE AND HAVERING PRIMARY CARE |TRUST: INTERIM EVALUATION}

M. Halter' ${ }^{1}$ S. Griffiths' ${ }^{2}$, J. Stevens ${ }^{2}$. 'London Ambulance Service NHS Trust, UK; ${ }^{2}$ Havering Primary Care Trust, UK

Introduction: The desire to deliver appropriate emergency care, the emphasis on A\&E and admission avoidance and on new professional roles led a PCT and ambulance service to pilot a district nurse and paramedic response team for 999 'category $\mathrm{C}^{\prime}$ calls.

Aim: To evaluate the impact of the team on:

- Ambulance performance

- Access to care pathways and health outcomes

- Patients

- Cost

Methods: Times, case type, treatment, and disposal are collected from ambulance service and district nursing patient records.

Patients are telephoned with a satisfaction and outcome questionnaire. Service costs are collected.

Results: 189 patients were attended in 6 months; averaging two per day. Workload in the area is considerably higher. Patients' averaged 70 years old. The largest patient groups are those with a 'fall', minor illness, or minor traumatic injury. Median response time is 15 minutes and the team spend a slightly increased time on scene. A\&E attendance is very low at $19 \% .76 \%$ of those 'non conveyed' were referred on - falls clinic (6), intermediate care team (6), social services (23), district nursing (25) and GP (57). Questionnaires received a $64 \%$ response rate - although expecting an ambulance, satisfaction was high with response time $(96 \%)$, the team $(98 \%)$, and treatment $(95 \%)$. 61\% considered their problem resolved. Cost per case is high due to low utilisation, but emergency ambulance and A\&E avoidance have opportunity costs.

Study limitations:

- No clinical follow-up

- No comparator data.

Conclusion: These interim results suggest the experience of the team for patients is very positive and access to care pathways is high. However, low utilisation reduces current cost effectiveness. This issue, and clinical review, are being addressed in the continuing pilot.

\section{IDENTIFICATION OF LOW PRIORITY EMERGENCY (999) CALLS TO THE AMBULANCE SERVICE SUITABLE FOR TELEPHONE ASSESSMENT AND ADVICE}

J. Turner ${ }^{1}$, H. Snooks ${ }^{2} .{ }^{1}$ Medical Care Research Unit, University of Sheffield, UK; ${ }^{2}$ The Clinical School, University of Wales Swansea, UK

Background: Telephone assessment and advice may provide more appropriate care to 999 callers who do not need an emergency ambulance response, as well as freeing up resources to respond to more serious cases.

Objective: To test the feasibility of passing low priority calls to NHS Direct for further assessment.

Methods: NHS Direct nurse advisors were introduced into an ambulance service in November 2002 between 08.00 and 22.00, 7 days a week. A list of priority dispatch codes potentially suitable for nurse assessment was identified. For a 5-month period, callers falling within these codes were passed to a nurse advisor, and the ambulance was stopped.

Results: 131318999 calls were received by the service within the study period, with 12070 falling within the designated dispatch codes. 2614 calls were passed to the nurse. $1770(67.7 \%)$ were returned to the ambulance service, although only 607 were coded as needing a 999 response; other reasons included: transport required $(n=620)$, patient request $(n=267)$, inappropriate (public place/caller not with patient) $(n=30)$, technical failure $(n=48)$. Pass back of calls was closely associated with patient's age: $0-25$ years, $52.6 \% ; 51-75$ years, $71.5 \%$; $76-100$ years, $80.3 \%$ ( $p<.001)$.

Conclusions: A high rate of return of 999 calls transferred to nurse advisors back to the ambulance service was found in this preliminary study. Refining the dispatch codes for transfer and setting up alternatives to the 999 response for calls returned need to be tested in order to maximise the potential for this initiative to be cost-effective.

\section{THE PARAMEDIC ASSESSMENT OF CARDIAC} EMERGENCIES (PACE) STUDY

A. Walker' , A. Taylor ${ }^{2}$, J. Humphrey ${ }^{2}$, A. Khan ${ }^{2}$, I. Barlow², S. Parnell ${ }^{2}$, C. Hague $^{2}$, G. Johnson' 2 , T. Hassan ${ }^{2}$. Mid Yorkshire Trust, UK; ' Emergency and Cardiology Departments, Leeds Teaching Hospitals NHS Trust, UK

Objective: Thrombolysis is a core intervention in modern emergency medicine and a variety of methods to reduce delays have been studied. The NSF for Coronary Heart Disease targets are for thrombolysis within 1 hour of the call for help and within 30 minutes of the hospital "door to needle". We assessed the impact of prehospital 12 lead ECGs by paramedics for patients with suspected cardiac chest pain in the Leeds Metropolitan area.

Methods: Paramedics were equipped with 12 lead ECG capability after a 4-day training programme. The patients were allocated to a 3 lead (control) or 12 lead ECG (PACE) by week day.

Outcomes:

1. Call to needle times

2. Door to needle times

3. Paramedic diagnostic accuracy

4. Drug treatment received by patients

5. Journey times/geography

Results: 436 patient were recruited, 98 AMls (46 in PACE arm, 52 in control arm)

Median Call to needle time: 62 minutes (PACE) $\vee 70$ minutes (control) $(p=0.18)$

Median Door to needle time: 15 minutes (PACE) v 23 minutes (control) $(p=0.12)$

Median pre-hospital time: PACE 45 minutes $v$ Control 44 minutes

Thrombolysed within 20 minutes of arrival: $69 \%$ (PACE) $\vee 44 \%$ (control)

Thrombolysed within an hour of call: $41 \%$ (PACE) $\vee 29 \%$ (control)

Paramedic diagnosis of STEMI: Sensitivity $95 \%$, Specificity $90 \%$

There were no differences in drug administration and no geographical clustering between the two groups.

Problems: Sample size.

Conclusions:

- Introducing a 12 lead ECG to prehospital care reduces overall thrombolysis times

- Increase in call to door time offset by gains in door to needle time

- Targets still difficult to achieve

- A prehospital ECG with paramedic interpretation alone is insufficient to accurately diagnose AMI for thrombolysis

- Alternative support systems such as telemetry should also be considered

- In the Leeds area patients in all zones may potentially benefit from prehospital thrombolysis

\section{PATIENT AND CALLER VIEWS OF COMMUNITY FIRST} RESPONDER SCHEMES

J. Turner ${ }^{1}$, Y. Owen ${ }^{2} .{ }^{1}$ Medical Care Research Unit, University of Sheffield, UK; ${ }^{2}$ Lincolnshire Integrated Voluntary Emergency Service (LIVES), UK

Background: A number of ambulance services have introduced community first responder schemes to help provide a faster response to life-threatening 999 calls in rural areas. This study assessed acceptability of and patient satisfaction with a community first responder service: the Lincolnshire Integrated Voluntary Emergency Service (LIVES). 
Methods: A postal questionnaire was developed to assess the views of users of the ambulance service who also received a LIVES response. Satisfaction with different aspects of the service were measured. These included:

- The 999 telephone call

- The LIVES response

- The ambulance service response

A total of 300 questionnaires were sent out to consecutive callers to the ambulance service who were allocated a LIVES response. A single mailing was carried out with no reminders.

Results: 180 questionnaires were returned giving a response rate of $60 \%$.

Callers reported a positive experience in terms of the handling of their 999 call. In $81 \%$ of cases the LIVES response arrived first at the scene. $87 \%$ of respondents thought LIVES arrived very promptly compared to $63 \%$ for the ambulance response. There was a high level of satisfaction with the LIVES response with respondents reporting strong agreement that they were helpful $(85 \%)$, professional $(84 \%)$, and efficient (83\%). Only $1.9 \%$ reported LIVES as not helpful. The LIVES response, call handling and the ambulance service all scored $>80 \%$ strongly agreeing on questions about satisfaction with the service. $75 \%$ strongly agreed that the local community providing medical help was acceptable and $91 \%$ thought the scheme important.

Conclusions: Users of the ambulance service showed a high level of satisfaction with both the first response service and the ambulance service. Characteristics of ambulance and LIVES personnel were highly rated and there were low numbers of individuals indicating any dissatisfaction. The principle of providing a voluntary medical response in rural communities seems to be acceptable to the public. 